Originally published as:

J. Schmidt-Chanasit, K. Tenner-Racz, D. Poppert, P. Emmerich, C. Frank, C. Dinges, R. Penning, A. Nerlich, P. Racz and S. Günther.

Fatal dengue hemorrhagic fever imported into Germany. (2012) Infection, 40, pp. 431-443.

DOI: $10.1007 / \mathrm{s} 15010-011-0208-3$

This is an author manuscript.

The definitive version is available at: http://link.springer.com 


\title{
Fatal dengue hemorrhagic fever imported into Germany
}

\author{
J. Schmidt-Chanasit ${ }^{1}$, K. Tenner-Racz ${ }^{2}$, D. Poppert ${ }^{3}$, P. Emmerich ${ }^{1}$, C. Frank ${ }^{4}$, C. Dinges ${ }^{5}$, \\ R. Penning ${ }^{5}$, A. Nerlich ${ }^{6}$, P. Racz ${ }^{2}$ and S. Günther ${ }^{1}$ \\ (1) Department of Virology, Clinical Virology Laboratory, Bernhard Nocht Institute for Tropical \\ Medicine, Bernhard-Nocht-Strasse 74, 20359 Hamburg, Germany \\ (2) Department of Pathology, Bernhard Nocht Institute for Tropical Medicine, Hamburg, Germany \\ (3) Academy for Psychosomatic Medicine and Psychotherapy, Munich, Germany \\ (4) Robert Koch Institute, Berlin, Germany \\ (5) Department of Forensic Medicine, University of Munich, Munich, Germany \\ (6) Department of Pathology, Klinikum Bogenhausen, Munich, Germany
}

Corresponding Author: J. Schmidt-Chanasit, Email: jonassi@gmx.de

\begin{abstract}
Dengue virus (DENV) is an arthropod-borne virus (family Flaviviridae) causing dengue fever or dengue hemorrhagic fever. Here, we report the first fatal DENV infection imported into Germany. A female traveler was hospitalized with fever and abdominal pain after returning from Ecuador.

Due to a suspected acute acalculous cholecystitis, cholecystectomy was performed. After cholecystectomy, severe spontaneous bleeding from the abdominal wound occurred and the patient died. Postmortem analysis of transudate and tissue demonstrated a DENV secondary infection of the patient and a gallbladder wall thickening (GBWT) due to an extensive edema.
\end{abstract}

\section{Introduction}

Dengue virus (DENV) is an arthropod-borne virus (arbovirus) within the family Flaviviridae and consists of four serotypes. DENV is able to infect humans and cause dengue fever or dengue hemorrhagic fever (DHF). There are 50-100 million cases of human DENV infections each year, of which $\sim 500,000$ are severe life-threatening infections [1,2]. DHF is more likely to occur in patients who have secondary DENV infections [3].

The majority of DENV infections is diagnosed in South-East Asia and South America. Very recently, the first autochthonous DENV infection acquired in South-East Europe was diagnosed [4]. DENV infections have been reportable in Germany since 2001, according to the Federal Protection against Infection Act [5]. From 2001 to 2010, a mean of 227 imported cases were reported per year (incidence $0.28 / 100,000$ ), with a maximum of 595 cases in 2010. Here, we describe the first fatal DENV infection imported into Germany. 


\section{Case report}

In 2009, a 21-year-old female was referred to an emergency room of a university hospital in Bavaria, Germany, with abdominal pain, fever $\left(>40^{\circ} \mathrm{C}\right)$, severe thrombocytopenia $\left(17,000\right.$ platelets $\left./ \mathrm{mm}^{3}\right)$, and elevated liver enzymes (aspartate transaminase 1,444 U/l, reference $<52 \mathrm{U} / \mathrm{l}$; alanine transaminase $834 \mathrm{U} / \mathrm{l}$, reference $<52 \mathrm{U} / \mathrm{l})$. Sonographic investigation of the abdomen revealed gallbladder wall thickening (GBWT). The patient had returned to Germany from Santo Domingo de los Colorados in Ecuador 8 days previously.

Due to a suspected acute acalculous cholecystitis and associated sepsis, cholecystectomy was performed 3 days after admission. Shortly after cholecystectomy, the clinical situation deteriorated rapidly and severe spontaneous bleeding from the abdominal wound occurred. Therefore, 34 units of fresh frozen plasma, 2 units of thrombocytes, and 29 units of packed erythrocytes where given. Severe bleeding continued despite intensive care, and the patient died in the evening of the 11th day of her illness.

Postmortem analysis of transudate and tissue demonstrated a DENV infection of the patient. Analysis of the transudate with an immunofluorescence assay (IFA) for DENV revealed an IgG titer of 1:2,560 and an IgM titer below 1:20. In addition, the serum sample was tested positive for DENV NS1 antigen. Real-time reverse transcription polymerase chain reaction (RT-PCR) for DENV was performed with transudate, liver, lung, spleen, heart, kidney, and brain specimens.

A positive result was obtained with liver, lung, and kidney. The PCR result was confirmed by sequencing the PCR product, which revealed a DENV serotype 1 infection. Attempts to isolate DENV in cell culture were not successful. Histopathological and immunohistochemical examinations were performed on paraffin sections. The liver showed marked fatty degeneration and small petechial hemorrhages. Small foci of hepatocellular necrosis were seen, mostly midzonal. Councilman bodies were present.

The wall of the gallbladder was thickened due to an extensive edema and cellular infiltration, mainly of lymphocytes. Immunohistochemical staining for NS1 of DENV 1 revealed virus antigen in some necrotic hepatocytes and Councilman bodies (Fig. 1). Focal positive staining was also seen in the gallbladder epithelium and in alveolar macrophages (Fig. 1). The postmortem findings are consistent with a secondary DENV infection, because of the simultaneous detection of DENV-specific IgG and RNA in the absence of IgM.

\section{Discussion}

In Germany, imported DHF cases are rare $(0.26 \%$ of imported DENV infections) and, until now, a fatal DENV infection was never recorded. Because of the thrombocytopenia and coagulation dysfunction in most DHF cases, it is difficult to obtain tissue samples for immunohistochemical analysis and, therefore, this analysis is often based on samples from fatal cases. In line with our results (Fig. 1), it was demonstrated that involvement of the liver, especially during DHF, may cause hepatocellular necrosis in the midzonal area, and that DENV antigen can be detected in Councilman bodies [ $\underline{6}-\underline{8}]$. Moreover, reticular GBWT has previously been demonstrated in severe DHF cases and is used as a reliable sign of plasma leakage [ 9$]$.

Therefore, reticular GBWT should not be considered as an acalculous cholecystitis [9], as in our case. However, the DENV infection of our patient could have been easily diagnosed with serological rapid tests, as previously demonstrated for other patients with imported DENV infections [10]. This fatal case has implications for the management of travelers with febrile illness returning from DENVendemic countries: DENV infection should be suspected in travelers presenting with fever and acute abdomen, and a surgical intervention in such patients should be postponed until a DENV infection is excluded. 


\section{Ethical standards}

The experiments comply with the current laws of Germany.

\section{Conflict of interest}

The authors declare that they have no conflict of interest. 


\section{References}

1. Allwinn R. Significant increase in travel-associated dengue fever in Germany. Med Microbiol Immunol. 2011;200:155-9.

2. Gubler DJ. Epidemic dengue/dengue hemorrhagic fever as a public health, social and economic problem in the 21st century. Trends Microbiol. 2002;10:100-3.

3. Whitehorn J, Farrar J. Dengue. Br Med Bull. 2010;95:161-73.

4. Schmidt-Chanasit J, Haditsch M, Schoneberg I, Gunther S, Stark K, Frank C. Dengue virus infection in a traveller returning from Croatia to Germany. Euro Surveill. 2010;15. pii: 19677.

5. Dreesman J, Benzler J. Infectious disease surveillance based on the Protection against Infection Act in the German public health sector. Bundesgesundheitsblatt Gesundheitsforschung Gesundheitsschutz. 2005;48:979-89.

6. Couvelard A, Marianneau P, Bedel C, Drouet MT, Vachon F, Hénin D, Deubel V. Report of a fatal case of dengue infection with hepatitis: demonstration of dengue antigens in hepatocytes and liver apoptosis. Hum Pathol. 1999;30:1106-10.

7. Huerre MR, Lan NT, Marianneau P, Hue NB, Khun H, Hung NT, Khen NT, Drouet MT, Huong VT, Ha DQ, Buisson Y, Deubel V. Liver histopathology and biological correlates in five cases of fatal dengue fever in Vietnamese children. Virchows Arch. 2001;438:107-15.

8. Seneviratne SL, Malavige GN, de Silva HJ. Pathogenesis of liver involvement during dengue viral infections. Trans R Soc Trop Med Hyg. 2006;100:608-14.

9. Oliveira GA, Machado RC, Horvat JV, Gomes LE, Guerra LR, Vandesteen L, Oliveira FT, Lousada NS, Moreira-Silva S, de Fatima Ceolin M. Transient reticular gallbladder wall thickening in severe dengue fever: a reliable sign of plasma leakage. Pediatr Radiol. 2010;40:720-4.

10. Schilling S, Emmerich P, Günther S, Schmidt-Chanasit J. Dengue and Chikungunya virus coinfection in a German traveller. J Clin Virol. 2009;45:163-4. 


\section{Figure}

Figure 1 Immunohistochemical analysis of the liver, gallbladder, and lung with the dengue virus (DENV) serotype 1-specific murine monoclonal antibody 15F3 (ATCC HB47) directed against NS1 antigen. The arrows indicate cells or areas with positive staining.
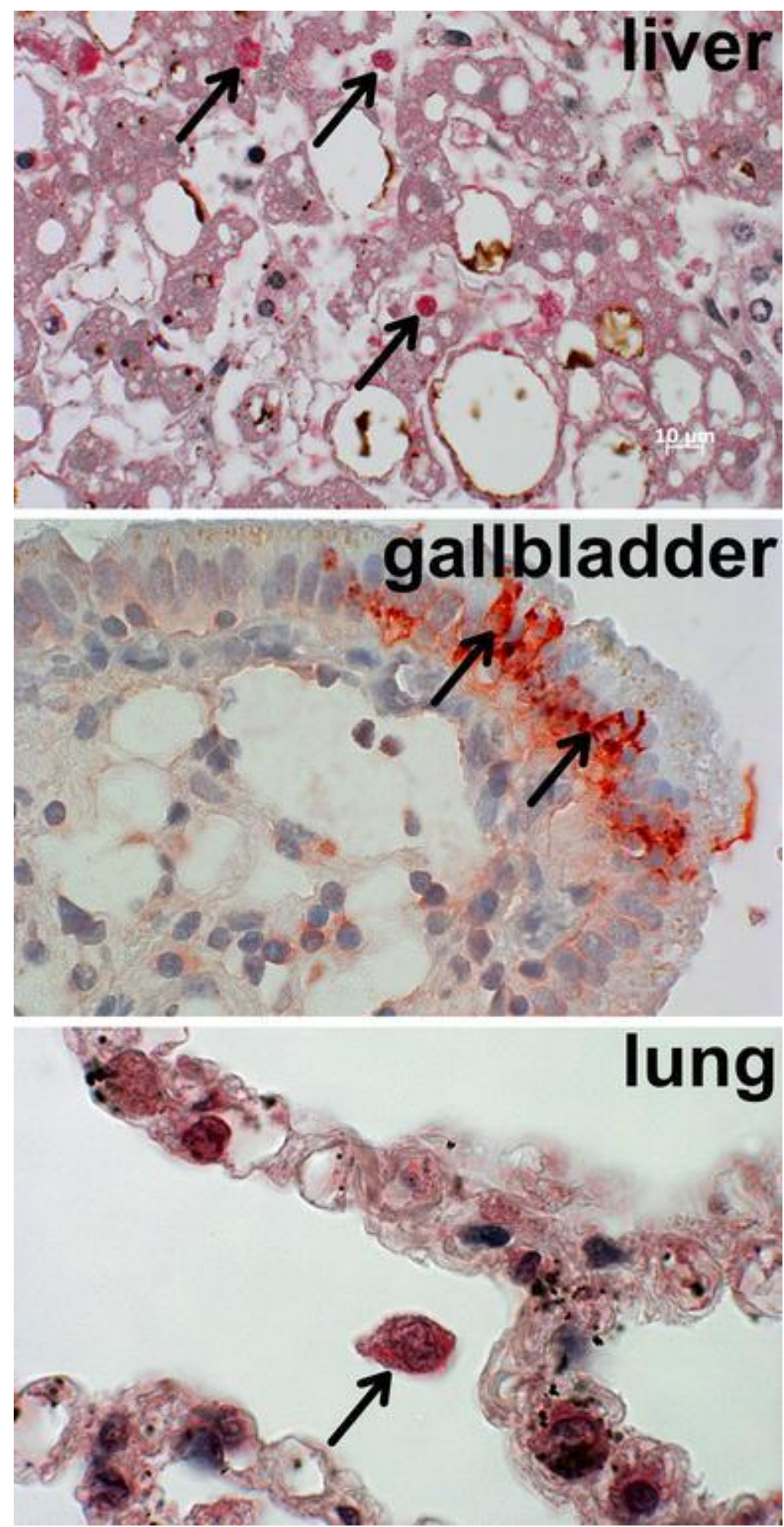